\title{
Advanced Propulsion Concepts For Orbital Transfer Vehicles
}

L. P. Cooper

Lewis Research Center

Cleveland, Ohio

Prepared for the

Nineteenth Joint Propulsion Conference cosponsored by the AIAA, SAE, and ASME

Seattle, Washington, June 27-29, 1983

\section{N/Sก}

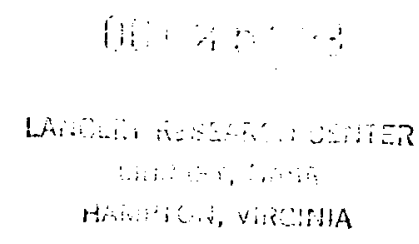




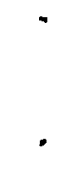


L. P. Cooper

National Aeronautics and Space Administration

Lewis Research Center

Cleveland, Ohio 44135

\section{Abstract}

Studies of the United States Space Transportation System show that in the mid-to-late 1990s expanded capabilities for Orbital Transfer Vehicles (OTV) will be needed to meet increased payload requirements for transporting materials and possibly men to geosynchronous orbit. NASA is conducting a technology program in support of an advanced propulsion system for future OTVs. This program is briefly described with results to date of the first program element, the "Conceptual Design and Technology Definition" studies.

\section{Introduction}

This paper reviews ongoing NASA sponsored efforts on advanced propulsion systems for Orbital Transfer Vehicles (OTV) and presents an overview of the "Conceptual Design and Technology

Definition" studies.

Analyses of potential space missions 1,2 for the 1990's indicate that individual payload requirements for transfer between low earth orbit and high or geosynchronous orbit during the 1990 's will be increasing. In addition, the total annual payload mass will increase substantially and manned missions will be undertaken. Currently available upper stage vehicles such as the Inertial Upper Stage and Centaur have not been designed to satisfy manned requirements of the late 1990's.

Current NASA Plans ${ }^{3}$ indicate that manned orbital support platforms will likely become operational during the 1990 's. The presence of a platform to support Orbital Transfer Vehicles would greatly increase flexibility to perform projected missions. OTV could be based at the space platform and mated to payloads on orbit, thereby alleviating payload length and weight limitations of the Space Shuttle.

Another factor influencing 1990's Orbital Transfer Vehicles will be the probable implementation of aeroassisted maneuvering to return from high earth orbits to low earth orbit. 4 This concept, as illustrated in Fig. 1, could substantially increase payload capability by reducing propellant requirements for the retroburn. The inaneuver uses the drag induced by the earth's atmosphere to reduce the OTV velocity. In addition, this maneuver could be used to perform orbital plane changes to accommodate payload placement or rendezvous with a support platform.

Another important facet of the 1990's space program which would impact the OTV propulsion system are manned missions. High reliability will be critical for these missions. In the past, this reliability has been accomplished through design margins and by system redundancy. The latter has attendant complexity and both increase weight. The criteria for man rating of future orbital Transfer Vehicles is unresolved and requires investigation.
Finally, versatility is a desirable feature for the advanced OTV. Manned missions, planetary missions, and Low G Transfer missions are all anticipated in the 1990's.

The projected propulsion system requirements for an OTV in the late 1990's will of course be influenced by the assumed missions and their frequency, the Shuttle capabilities in terms of payload weight and volume and the basing mode of the 0TV. The space based, manned, aero assist and Low $G$ Transfer missions are the technology driver missions for the OTV propulsion concepts. Their impact will be felt as requirements for enhanced reliability, reusability, life and on-orbit maintenance as well as improved performance and versatility. With these considerations in mind, NASA has established an Advanced OTV Propulsion Technology Program. This program, which was initiated in 1981, will now be described.

\section{Advanced OTV Propulsion Technology}

The objective of this program is to establish by 1990 the technology base for high performance, multiple restart, variable thrust, orbital transfer propulsion systems which could be man rated, space or ground based, and compatible with aeroassisted maneuver vehicles.

As a precursor to the development of an advanced OTV propulsion system, this program will enable incorporation of the latest technology into the advanced engine while providing a low risk and minimum cost approach.

\section{Propulsion System Characteristics}

The required propulsion system characteristics for this program are listed in table 1 and include cryogenic hydrogen-oxygen propellants and a vacuum thrust level of 10000 to 25000 pounds. These parameters were selected to be compatible with a manned sortie to geosynchronous orbit. The mixture ratio design point of 6.0 and range of 5.0 to 7.0 was selected based upon thrust chamber cooling and life as well as propulsion system/ vehicle length and weight considerations. The thrust vector control requirement of $+6^{\circ}$ was based upon a survey of potential payloads, vehicles, and missions. The start cycle with propulsive dumping of propellant reflects the philosophy of maximizing the usefulness of propellant resources.

In addition to these specific required characteristics, this technology program has established several goals reflecting characteristics desirable for an advanced engine. These goals are listed in table 2. In total they represent a set of highly ambitious characteristics for an advanced engine and were established as technical challenges to generate options and tradeoffs since all goals may not be achievable singularly or concurrently. For example, the vacuum specific impulse goal, $520 \mathrm{lbf}-\mathrm{sec} / 1 \mathrm{bm}$, is the maximum value 
without phase change of the exhaust for a hydrogen-oxygen engine.

Selection of a 30:1 throttling range reflects the versatility needed to perform Low $G$ orbit transfer missions as well as to provide thrust variability for aeromaneuvering. Low net positive suction head (NPSH goal-0. ft-ibf/lbm) for the hydrogen and oxygen turbomachinery is desirable to decrease propellant residuals, tank weight, and pressurization requirements as well as to avoid pump cavitation.

Propulsion system weight is generally a small portion of the total vehicle. However, it is generaliy desirable to minimize the propulsion weight since it directly trades off against payload delivery. The goal was established at 360 pounds based on earlier studies ${ }^{5}$ of advanced OTV engines. The minimized propulsion system stowed length goal of 40 inches was derived from consideration of length constraints in the Space Shuttle payload bay. Minimum length is particularly important since the majority of projected shuttle payloads are volume rather than mass limited.

For a man-rated OTV, high reliability is required. A number of possible vehicle and propulsion system configurations are possible depending upon the expected component reliability. A reliability goal of 1.0 has been established for the advanced engine.

Finally, reusable OTVs will require periodic maintenance. If the OTV is ground based, this will naturally be performed on the return to earth. However, if the OTV is space based, the maintenance would be performed in space. The duration, frequency, and complexity of these maintenance tasks are important issues. By maximizing service-free life and time between overhauls, the down time and cost of maintenance should be reduced for both ground and space-based OTVS.

\section{Approach}

The advanced OTV propulsion technology program has been structured around a projected need date for an advanced engine of 1995 with a development program beginning in 1990 . The technology program is composed of three elements:

1. Conceptual Designs \& Technology Definition consisting of study efforts to conceptually define advanced OTV propuision systems and to identify, screen, and propose advanced technology concepts at the subcomponent, component, and propulsion system levels which would benefit the future OTV propulsion system.

2. Exploratory Research consisting of analytical and experimental efforts to evaluate advanced technology concepts critical to the success of future 0TV propulsion systems.

3. Critical Component Technology to experimentally verify the technology readiness of critical components.

A programmatic schedule is shown in table 3 . Conceptual Design \& Technology Definition was initiated in 1981 with three rocket engine manufacturers and will continue throughout the program, generating technology concepts for advanced propulsion systems. Exploratory Research has been initiated in 1983 while the Critical Component Technology will begin in 1985 .
Conceptual Designs \& Technology Definition

Studies $6-8$ were initiated in 1981 with Aerojet Liquid Rocket Company, United Technologies Corporation-Pratt \& Whitney Aircraft Group, and Rockwell International-Rocketdyne Division, to define propulsion concepts for an advanced orbit Transfer Vehicle and to identify the technologies which would be required to demonstrate technology readiness for a Design, Development, Test and Engineering (DDTeE) program in the early 1990's. The concepts and technologies defined by each Contractor are presented below.

\section{Aerojet Liquid Rocket Company}

Propulsion Concept - A dual-propellant expander-cycle engine, sized for a nominal thrust level of 3000 pounds, was identified. The engine is throttlable over a 30:1 range with tank head idle mode producing 100 pounds of thrust and a 15:1 continuously throttlable pumped mode from 200 to 3000 pounds shrust. When applied to an advanced 0TV, a multiple engine installation as shown in Fig. 2 would be utilized to achieve the desired total thrust level. The engine's specific impulse of 482 seconds at a mixture ratio of 6.0 * would be obtained through utilization of a chamber pressure of 2000 psia and a nozzle area ratio of 1200:1. The engine's overall length is 77 inches, and the estimated engine weight is 126 pounds. When attached to the vehicle as shown in Fig. 2 a zero additional stage length penalty could be obEained if the area ratio were reduced to $400: 1$. A loss of approximately 5 seconds in specific impulse would result.

The flow schematic of the Aerojet concept is shown in Fig. 3. The key feature of the dual propellant expander cycle is that both hydrogen and oxygen are utilized to drive their respective turbopumps. This approach enables a higher chamber pressure to be obtained than with the conventional hydrogen expander cycle and also eliminates interpropellant seals in the oxidizer turbopump.

The critical technology issue to be resolved for this engine is the hazard of explosion or combustion of oxygen turbine materials exposed to the warm gaseous oxygen in the dual propellant cycle. Frictional rubbing or particle impact could produce disastrous results. A review of the litera-

Ref ture indicates that with careful material selection and design this potential problem can be overcome.

Turbomachinery - The oxidizer turbopump is a two stage centrifugal pump and two stage turbine design operating at 75000 revolution per minute (rpm) to produce oxidizer pressure of 4800 pounds per square inch absolute ( $p$ ia) at the design point thrust of 3000 pounds. The concept features a rigid rotor and stiff hydrostatic bearings so that the turbopump is at subcritical speed at all operating points. Turbopump life is enhanced by

\#projected specific impulse values will vary with the analysis method and assumptions employed. As of this date, the accuracy of these procedures has not been established for nozzle area ratios exceeding 400:1. Universal agreement does not exist regarding the correct procedures and assumptions for these high area ratios. Specific impulse values in this paper are best estimated by the Contractors. 
the hydrostatic bearing which minimizes rubbing. The turbine is driven by warm oxygen thereby eliminating the interpropellant seals required by hydrogen driven turbines.

The hydrogen turbopump is a four stage centrifugal pump-two stage turbine design operating at $200000 \mathrm{rpm}$ to produce 4800 psia hydrogen at the design operating point. The concept also utilizes hydrostatic bearings for long life and utilizes a partial admission on the turbine for high efficiency. The design approach enables the turbopump to operate subcritical over the entire operating range up to $200000 \mathrm{rpm}$.

Thrust Chamber - The combustion chamber is an annular design with a centerbody housing the oxidizer turbopump as shown in Fig. 4. This centerbody is regeneratively cooled with the oxygen used to drive the oxygen pump turbine. The outside walls of the chamber are regeneratively cooled with the hydrogen used to drive the hydrogen pump turbine. The design approach enables high surface area to be coupled with low heat flux to meet the total power requirements for high chamber pressure and yet provide a long life thrust chamber.

Nozzle - The design nozzle area ratio is 1200 which represents an exit diameter of approximately 30 inches. The nozzle is regeneratively cooled to an area ratio of 417:1 from which point the nozzle is radiation cooled. Either a refractory metal or a carbon composite will be utilized for this radiation cooled segment.

Controls \& Sensors - Aerojet has selected a closed-Toop system for eng ine control. Electronic and mechanical sensors monitor the engine's operation, and provide control feedback to maintain optimum performance and flexibility of the engine for the entire mission. Closed-loop control will be particularly important for a multiple engine installation where capability to operate with engines out while maintaining the selected thrust vector will be a complex task.

In addition to control sensors, diagnostic sensors will be incorporated in the engine to facilitate condition monitoring for maintenance.

Pratt \& Whitney

Propulsion Concept - A hydrogen expander cycle sized for a nominal thrust level of 15000 pounds with oxidizer preheating and hydrogen regeneration was selected. The concept is throttlable over $30: 1$ range with 3 discrete operating modes; tank head idle, pumped idle, and full thrust. As shown in Fig. 5 the concept has a chamber pressure of $1500 \mathrm{psia}$ and has a nozzle area ratio of 640:1 resulting in a specific impulse of 486 seconds at a mixture ratio of 6.0 . The total length of the concept is 120 inches, but can be packaged into a 40 -inch length by retracting the nozzle in 3 segments around the core. The concept has a weight of 450 pounds, which could be reduced by up to 30 pounds for a space-based OTV through simplifying the nozzle and deployment mechanisms.

The propellant flow schematic for this expander cycle engine is shown in Fig. 6. A unique element in this concept is the hydrogen cooled gears in the turbopump assembly which synchronize the oxygen and hydrogen pumps and drive low-speed inducers.

Turbomachinery - The oxidizer pump is a single-stage, shrouded, centrifugal design operating at $67390 \mathrm{rpm}$ to produce $2660 \mathrm{psia}$ outlet pressure. The hydrogen pump is a two-stage, centrifugal design operating at $150000 \mathrm{rpm}$ to prosluce 3905 psia out let pressure. A synchronizing zear is included between the pump shafts. Both pumps are fed by low-speed, axial-flow, inducer-type, boost pumps which are driven by a gear train off the oxidizer pump shaft. Two single-stage, axialflow, hydrogen turbines drive the turbopumps. The turbomachinery is designed to operate at subcritical speeds for all operating conditions. Thrust Chamber - The selected thrust chamber concept is regeneratively hydrogen cooled with a rippled wall design as shown in Fig. 7. This design, coupled with an advanced copper alloy, promises to provide a long life thrust chamber. Nozzle - For ground based OTV applications, the nozzle is an extendable 3 segment design providing an area ratio of 640:1 with an exit diameter of 64 inches. The first nozzle segment to 210:? area ratio is a tubular regeneratively cooled design, and the remaining segments are radiation cooled. Carbon-carbon composite is a leading candidate for these segements. When space based, this nozzle design and its extension/ retraction mechanism could be considerably simplified and the weight reduced by up to 30 pounds. Controls \& Sensors - The Pratt \& Whitney engine concept is open-Toop controlled. No engine operation information is fed back to affect the engine mixture ratio or thrust control. The key engine feature which enables open-loop control is the turbomachinery synchronizing gear which maintains turbopumb output at the established mixture ratio over the operating speed ranges. The philosophy behind selecting open-loop control lies in the tradeoff between simplicity and reliability vs. opportunities for optimizing performance during missions and to take corrective action in the event of degraded engine operation. Sensors required for the engine operation are minimized with open loop control system although diagnostic sensors to monitor engine operations will be incorporated as needed to facilitate maintenance requirements.

\section{Rocketdyne}

Propulsion Concept - A hydrogen expander cycle with oxidizer preheating and hydrogen regeneration was selected as the advanced propulsion concept. The concept was sized for 15000 pound thrust throttlable over a $30: 1$ in three discrete operating modes; tank head, pumped idle, and full thrust operation. As shown in Fig. 8, at a mixture ratio of 6.0 , the concept has a specific impulse of 492 seconds with a chamber pressure of 2000 psia and a three segment extendable nozzle of 1300:1 area ratio. With the nozzle retracted, the total length is 40 inches. The engine is est $i$ mated to weigh 435 pounds in the space-based configuration and 20 pounds more if ground based.

The propellant-flow schematic for this expander cycle is shown in Fig. 9. The unique feature of this concept is that the hydrogen turbomachinery operates above the $3 r$ critical speed.

Turbomachinery - The hydrogen pump is a four stage centrifugat design preceded by an inducer and driven by a two stage partial admission turbine. The turbopump has a hydrogen outlet pressure of $8000 \mathrm{psia}$ and operates at $178000 \mathrm{rpm}$. This speed, which is in excess of the third critical speed of the turbopump, represents a considerable challenge in transitioning through the lower 
critical speeds and avoiding subsynchronous whirl. The oxygen turbopump is a single stage centrigual design preceded by an inducer and driven by a single stage partial admission turbine. The turbopump has an outlet pressure of 3400 psia and operates at $56200 \mathrm{rpm}$. Both the oxygen and hydrogen turbopumps incorporate hydrostatic bearings for long life. Axial inducer type boost pumps feed tank propellants to each turbopump.

\section{Thrust Chamber}

Advanced heat extraction and 1 ife enhancement techniques are incorporated into the regeneratively hydrogen cooled thrust chamber design. The chamber has a series of longitudinal fins on the hot-gas side which increase surface areas as well as the local heat-transfer coefficient. On the coolant side of the chamber, fins have been incorporated to increase the surface area for transferring heat more effectively to the hydrogen coolant.

\section{Nozzle}

The concept utilizes a 1300:1 area ratio nozzle with an exit diameter of 78 inches which is regeneratively cooled to 575:1 area ratio and radiation cooled for the remainder. A carboncarbon composite is the candidate material for this latter portion. For ground based application, the nozzle would be divided into 3 segments of approximately 40 inches which would retract over the engine core. The nozzle extension/retraction mechanism could be simplified and possibly eliminated for a space-based OTV.

\section{Controls \& Instrumentation}

In order to maintain optimum performance and mission flexibility of the engine, a closed loop control system has been selected, although this introduces additional complexity and reliability requirements. Sensors will be included to monitor engine status with some being active control sensors while others would record flight information history for maintenance records.

\section{Discussion}

Shown in table 4 is a chart summarizing the key features of the three advanced OTV propulsion concepts. All utilize the expander power cycle rather than the staged combustion cycle. Pratt \& Whitney and Rocketdyne use the hydrogen expander cycle while Aerojet uses a hydrogen and oxygen expander cycle. By applying advanced technology, the chamber pressure of the simpler expander cycle can be increased so that performance is comparable with a staged combustion cycle engine of similar size while retaining superior life features of the expander cycle. All the engine concepts utilize high chamber pressure and large area ratio nozzles to obtain high performance.

The Aerojet single engine thrust level of 3000 pounds was selected to facilitate multiple engine installation, reflecting Aerojet's approach to reliability for a man-rated OTV. Similarly, the selection by the other Contractors of 15000 pounds thrust concepts reflects their current assessment of manned missions and man rating of OTVs. A single engine OTV is optimally sized at nominally 15000 pounds thrust for manned GEO missions and with suitable backup systems may represent a viable approach to man rating the vehicle. Hydrogen turbopump speeds are well beyond the state-of-the-art for all concepts. Each engine concept utilizes a different approach. Aerojet's turbopump has the highest operating speed. The design utilizes hydrostatic bearings for long life and stiff shaft appraoch to avoid operation above the first critical speed. Pratt \& Whitney's turbopump utilizes roller bearings and an extremely stiff shaft design to avoid the ist critical speed. Rocketdyne's turbopump design operates between the 3 rd and 4 th critical speed and could encounter subsynchronous whirl.

The oxygen turbopump of the Aerojet concept is oxygen driven while Pratt \& Whitney and Rocketdyne favor conventional hydrogen drive. oxygen drive presents some interesting options in engine packaging and eliminates interprope? lant seals. However, the metal ignition hazard must be carefully addressed. Only Pratt \& Whitney has gear driven turbomachinery. This approach simplifies control, but represents a considerable technology barrier to obtain long engine life and low maintenance.

For propulsion system control, Aerojet and Rocketdyne have selected closed-loop control to maintain flexibility for optimum mission performance and in Aerojet's case to enable multiple engine thrust vector control. Pratt \& Whitney has selected the open loop control method. All engine concepts would provide sufficient monitoring sensors to establish maintenance needs.

\section{Concluding Remarks}

NASA has a multi-element program to establish advanced engine technology for a future Orbit Iransfer Vehicle propulsion system. The program's objective is to provide the technology to enable a low risk and minimum cost design, development, test and engineering (DDT\&E) program for an advanced OTV engine to proceed in the early 1990's. Currently, three engine concepts and associated technologies have been evolved providing a range of options to satisfy future missions.

In general, the technologies can be grouped. by the major engine components of thrust chamber, turbomachinery, and nozzle. For the thrust chamber, the chief concerns are long life and increased energy extraction. Advanced materials and enhanced heat-transfer concepts have been identified. In the turbomachinery components, high rotational speeds raise life and performance concerns for the bearings, seals, and gears as well as concerns over material stress levels and fabrication. For the nozzle, extension. and retraction mechanisms, performance, and fabrication of very high area ratio lightweight nozzles are the technology issues.

The three engine concepts identified in these studies are only.baseline engines for defining technology needs for an advanced OTV engine. The identified technologies can generally be applied to engines in the 2500 to 25000 pound thrust range. The final engine may vary significantly from any one of the concepts. Technologies will continue to be identified and refined over the next several years. Particular emphasis will be placed on issues of the integration of the propulsion system with the Orbital Transfer Vehicle, criteria for man-rating 0TVs, requirements imposed 
by aeroassisted maneuvering, and implications of space basing and space maintenance.

\section{References}

1. NASA Marshall Space Flight Center, Preliminary Nominal Mission Model, (Revision 6, October 4, 1982).

2. NASA Marshall Space Flight Center, Preliminary OTV Mission Model, (Revision 2, February 27, 1980).

3. 01stad, W. B., "Targeting Space Station Technologies", Astronautics and Aeronautics, Vol. 21, No. 3, Mar. 1983, pp. 28-32.

4. Davis, E. E., "Future Orbital Transfer Vehicle Technology Study", NASA CR-3536, 1982.
5. Zachary, A. T., "Advanced Space Engine Preliminary Design," Rocketdyne Canoga Park, Ca., R-9269, Oct. 1973. NASA CR-121236.

6. A. Martinez, "Orbit Transfer Rocket Engine Technology Program," Vol. 2, Rocketdyne Division Rockwell International, Canoga Park, California, to be published. NASA CR-168158.

7. L. Schoenman, "Orbit Transfer Rocket Engine Technology Program," Vol. 2, Aerojet Liquid Rocket Company, Aerojet General, Sacramento, California, to be published. NASA CR-168157.

8. J. Brown, "Orbit Transfer Rocket Engine Technology Program," Vol. 2, Pratt Whitney Aircraft Group/GPD, United Technologies Corporation, West Palm Beach, Florida, to be published. NASA CR-168156. 


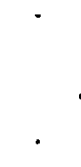


TABLE I. - REQUIRED ADVANCED OTV PROPULSION SYSTEM CHARACTERISTICS

\begin{tabular}{|l|l|}
\hline \multicolumn{1}{|c|}{ Characteristic } & \multicolumn{1}{|c|}{ Requirement } \\
\hline Propellants - fuel & Hydrogen \\
oxidizer & 0xygen \\
*Vacuum thrust (design point range) & 10000 to $25000 \mathrm{LBF}$ \\
Engine mixture ratio, 0/F (design point) & 6.0 \\
Engine mixture ratio range, 0/F & 5.0 to 7.0 \\
Propellant inlet temperature - Hydrogen & $37.8^{\circ} \mathrm{R}$ \\
oxygen & $162.7^{\circ} \mathrm{R}$ \\
Thrust vector control & $\pm 6.0^{\circ}$ \\
(square pattern) \\
Start cycle & Chilldown with propulsive dumping of \\
& propellants, engine start with pump \\
& inlets at propellent tank vapor \\
& pressure. \\
\hline *Vacuum thrust range may be obtained from either a single engine or multiple \\
engine configurations having total thrust within the specified range.
\end{tabular}

TABLE II. - ADVANCED OTV PROPULSION SYSTEM GOALS

\begin{tabular}{|l|r|}
\hline \multicolumn{1}{|c|}{ Characteristic } & \multicolumn{1}{|c|}{ Goal } \\
\hline Vacuum specific impulse, lbf-sec/lbm & 520 \\
Vacuum thrust throttle ratio & $30: 1$ \\
Net positive suction head, ft-lbf/lbm & \\
Hydrogen & 0 \\
Oxygen & 0 \\
Weight, lbm & 360 \\
Length (stowed), in. & 40 \\
Reliability & 1.0 \\
Service life & $500 / 20$ \\
Between overhauls, cycles/hours & $100 / 4$ \\
Service free, cycles/hours & \\
\hline
\end{tabular}


TABLE III. - ADVANCED OTV PROPULSION TECHNOLOGY PROGRAM SCHEDULE

Element

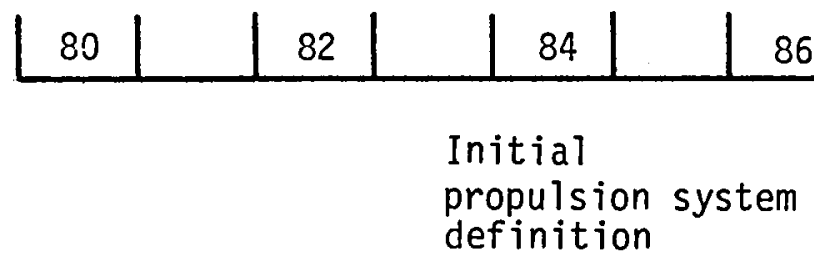

\section{Final}

propulsion system definition

Conceptual designs and

technology definition

Exploratory research

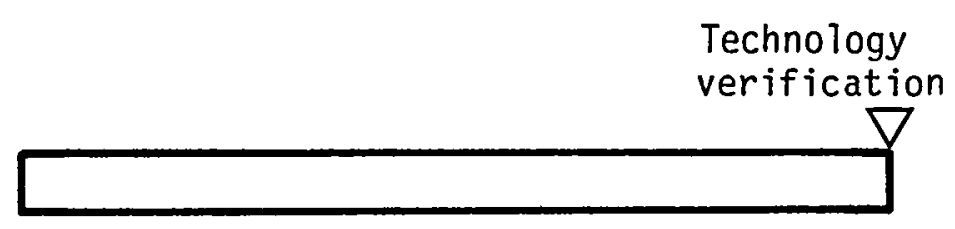

Critical component technology

Technology

Readiness

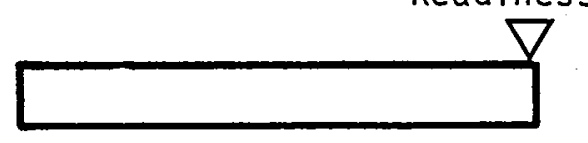

TABLE IV. - ADVANCED OTV PROPULSION SYSTEM CONCEPTS

\begin{tabular}{|l|l|l|l|}
\hline & \multicolumn{1}{|c|}{ Aerojet } & Pratt Whitney & Rocketdyne \\
\hline Thrust, 1bf & 3000 & 15000 & 15000 \\
Cycle & Expander $\mathrm{H}_{2}-02$ & Expander $\mathrm{H}_{2}$ & Expander $\mathrm{H}_{2}$ \\
Chamber pressure, psia & 2000 & 1500 & 2000 \\
Nozzle area ratio & $1200: 1$ & $640: 1$ & $1300: 1$ \\
Specific impulse, 1bf-sec/7bm & 482 & 486 & 492 \\
Turbomachinery speeds, rpm & 200000 & 150000 & 178000 \\
$\mathrm{H}_{2}$ & 75000 & 67390 & 56200 \\
$0_{2}$ & Closed loop & $0 p e n$ loop & Closed loop \\
Control & $30: 1$ step & $30: 1$ step & $30: 1$ \\
Throttlability & 2 step \\
Range & $(15: 1$ continous $)$ & discrete & discrete \\
Mode & \multicolumn{2}{|l}{} \\
\hline
\end{tabular}




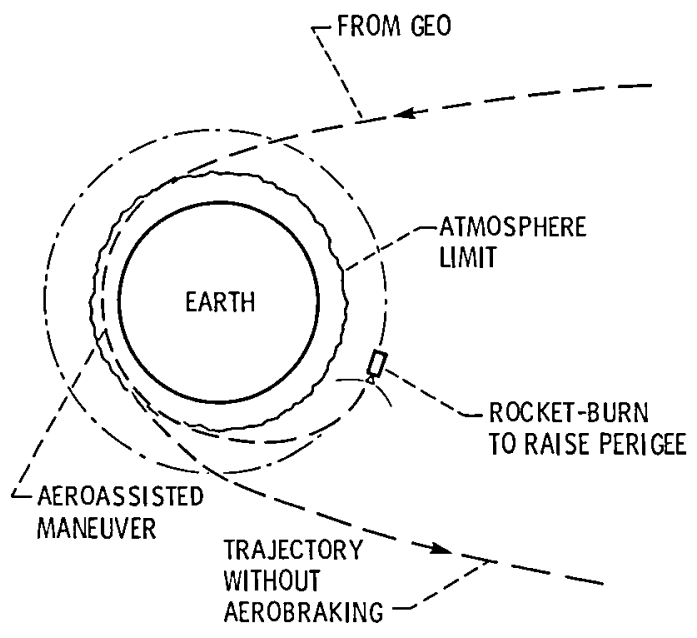

Figure 1. - Aero assisted vehicle maneuver.

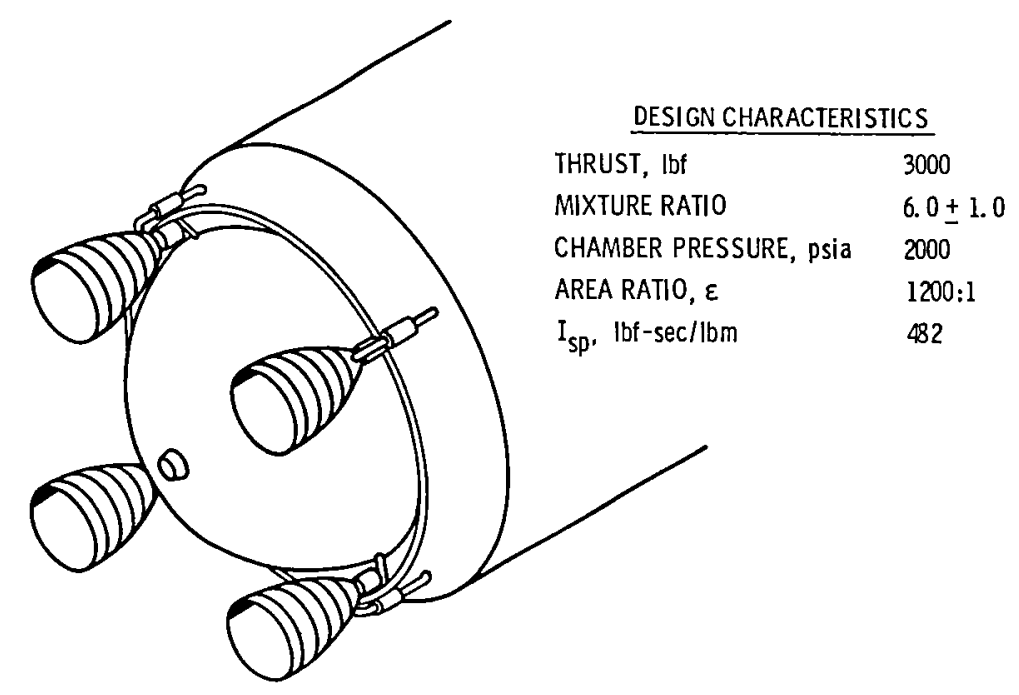

Figure 2 - Advanced OTV propulsion concept; Aerojet Liquid Rocket Company. 


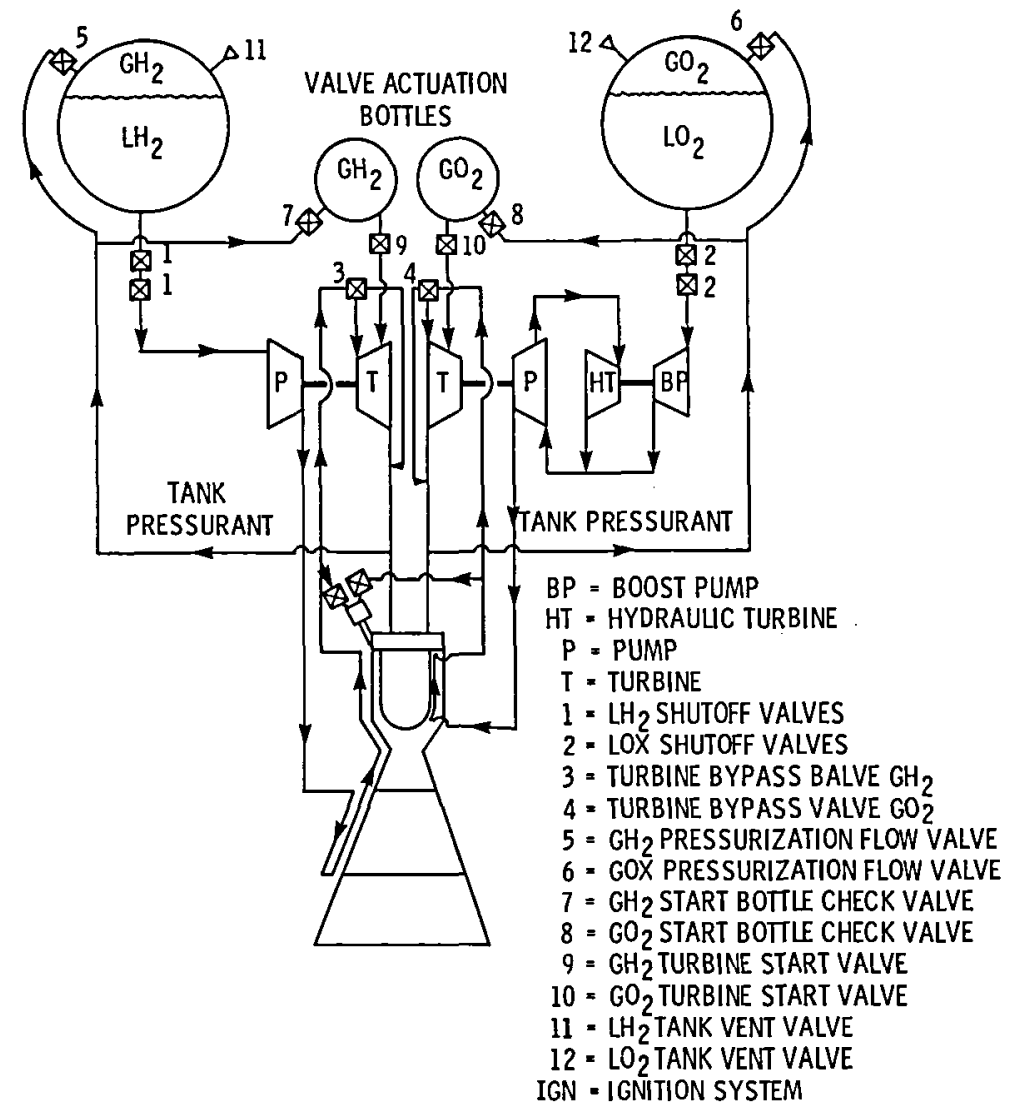

Figure 3. - Flow schematic; Aerojet advanced OTV propulsion concept

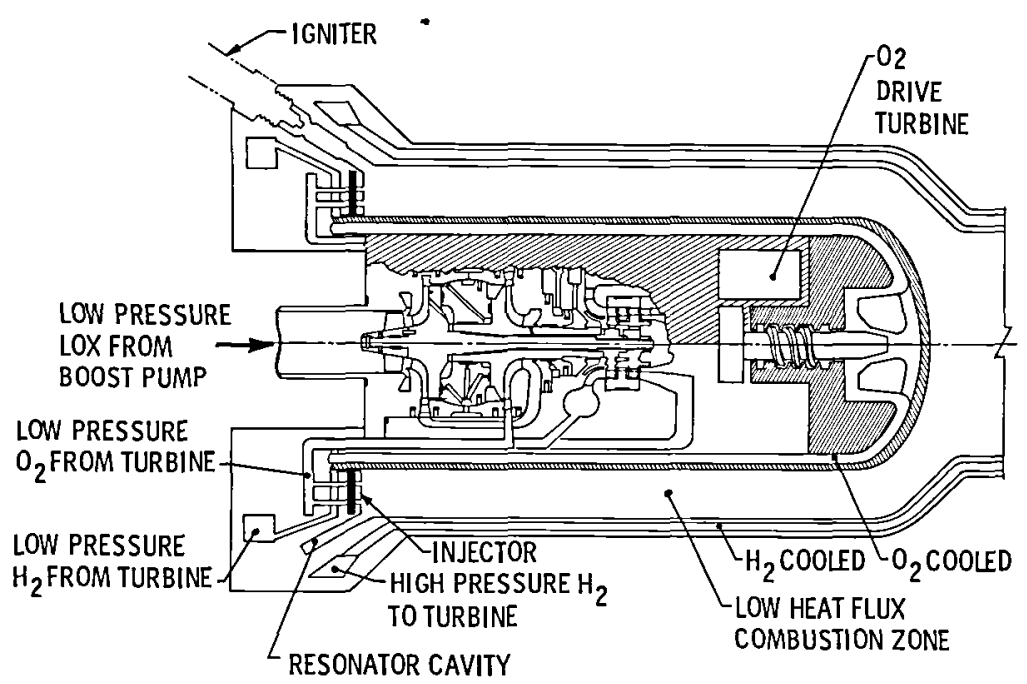

Figure 4 - Thrust chamber and oxygen turbopump; Aerojet advanced OTV propulsion concept. 


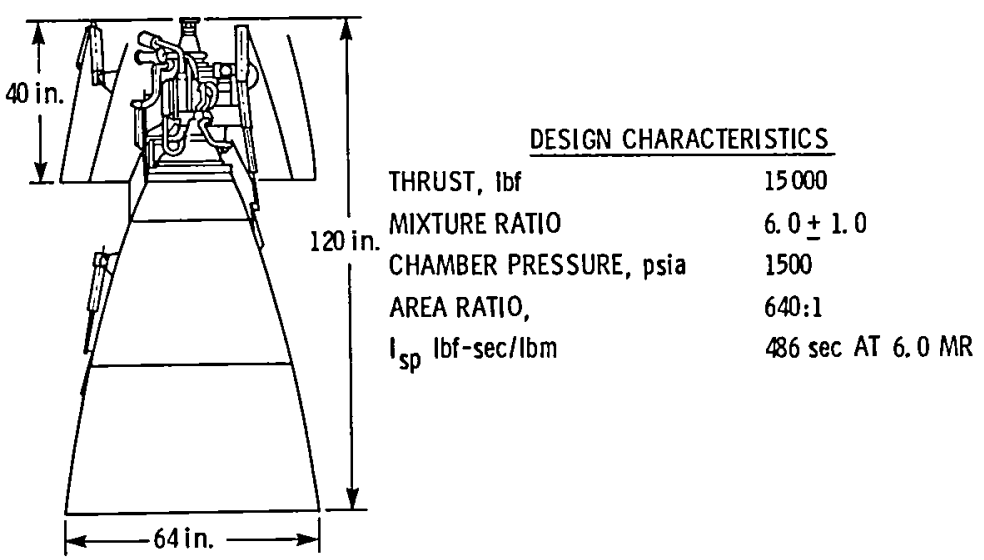

Figure 5. - Advanced OTV propulsion concept; Pratt \& Whiiney Aircraft Group. 


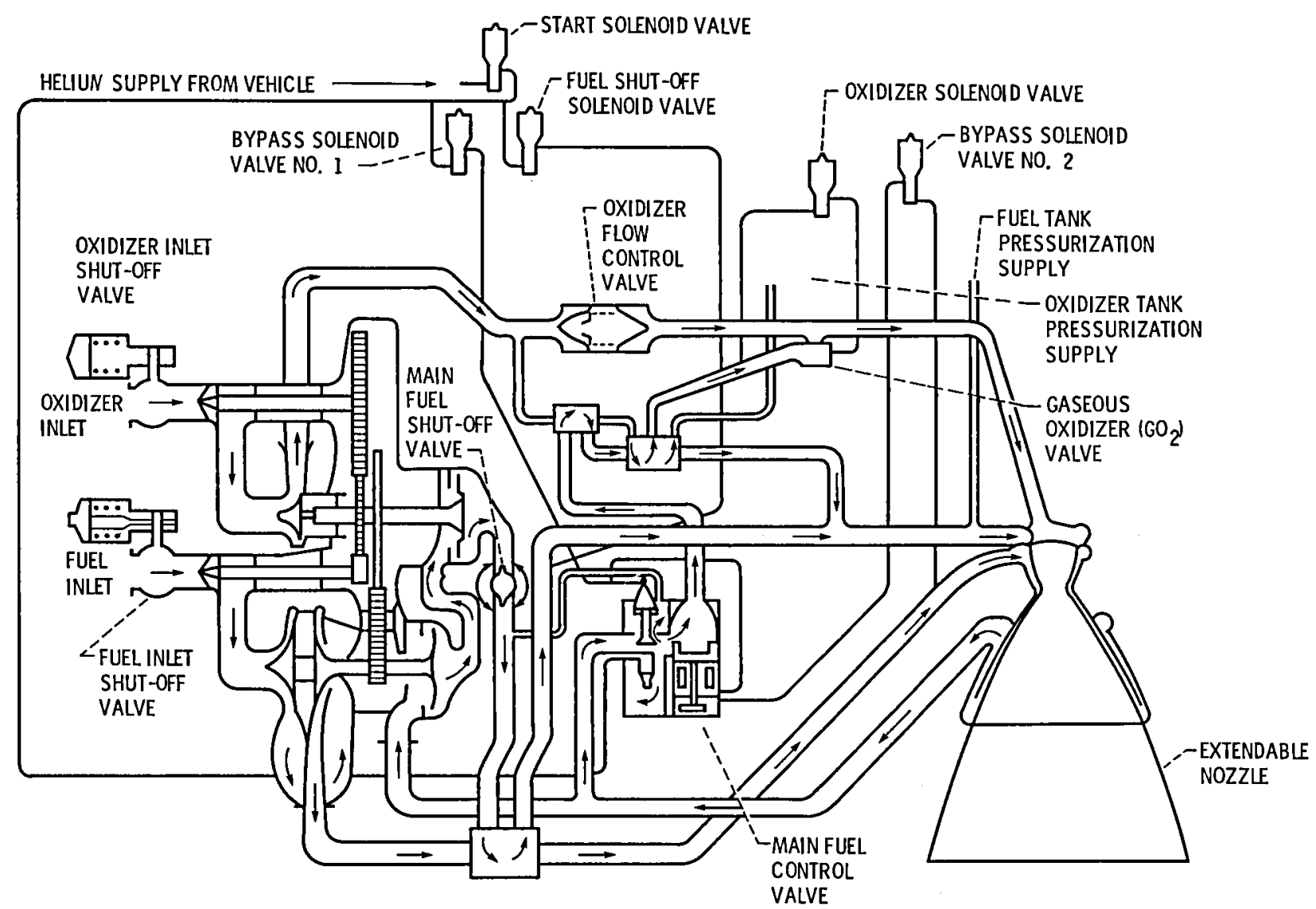

Figure 6. - Flow schematic; Pratt \& Whitney advanced OTV propulsion concept. 


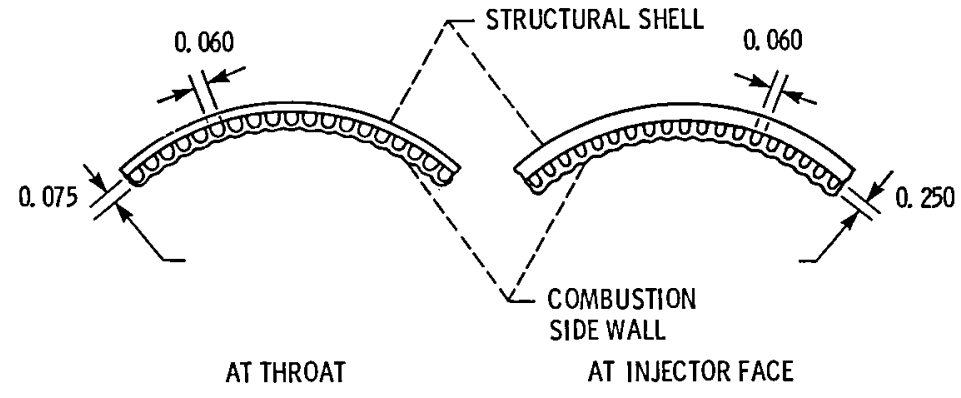

CHAMBER DIAMETER 5.06 in.

THROAT DIAMETER $253 \mathrm{in}$.

AXIAL LENGTH $15.00 \mathrm{in}$.

- 80 MILLED COOLING PASSAGE SLOTS VARY IN DEPTH ALONG AXIAL LENGTH

- ELECTRO FORMED OUTER WALL PROVIDES STRUCTURAL SHELL

Figure 7. - Thrust chamber, Pratt \& Whitney advanced OTV propulsion concept.

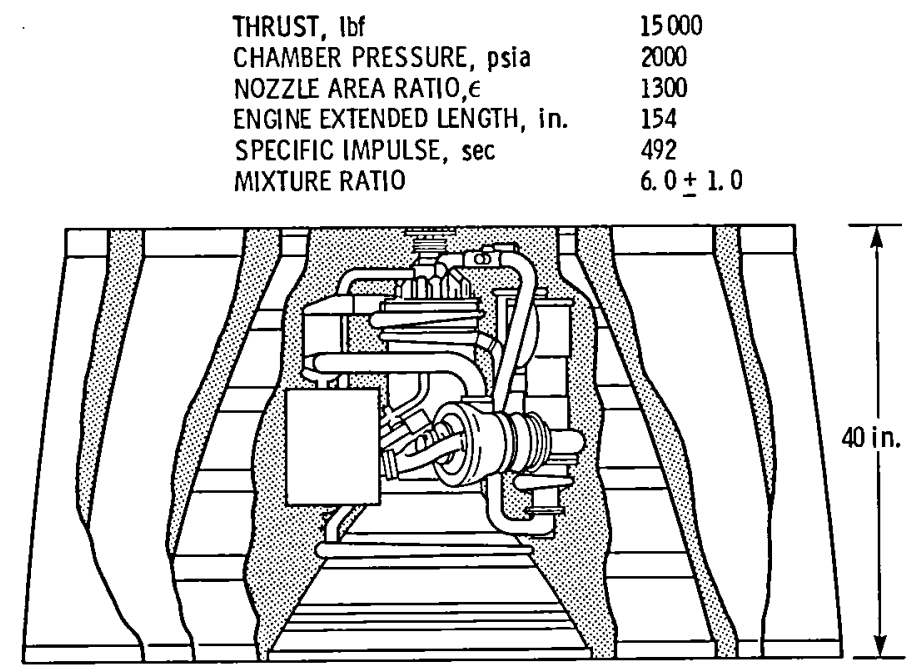

Figure 8. - Advanced OTV propulsion concept; Rocketdyne Division. 


\section{ENGINE CONTROLS}

(1) IFV - INLET FUEL VALVE

(2) IOV - INLET OXIDIZER VALVE

(3) MFV - MAIN FUEL VALVE

(4) MOV - MAIN OXIDIZER VALVE

(5) TBV - TURBINE BYPASS VALVE

(6) OTBV - OXIDIZER TURBINE BYPASS VALVE

(7) GOV - GASEOUS OXIDIZER VALVE

(8) DFV - DUMP FUEL VALVE

(A) FULLFLOW HYDRAULIC TURBINE

FOR LOW PRESSURE LOX PUMP

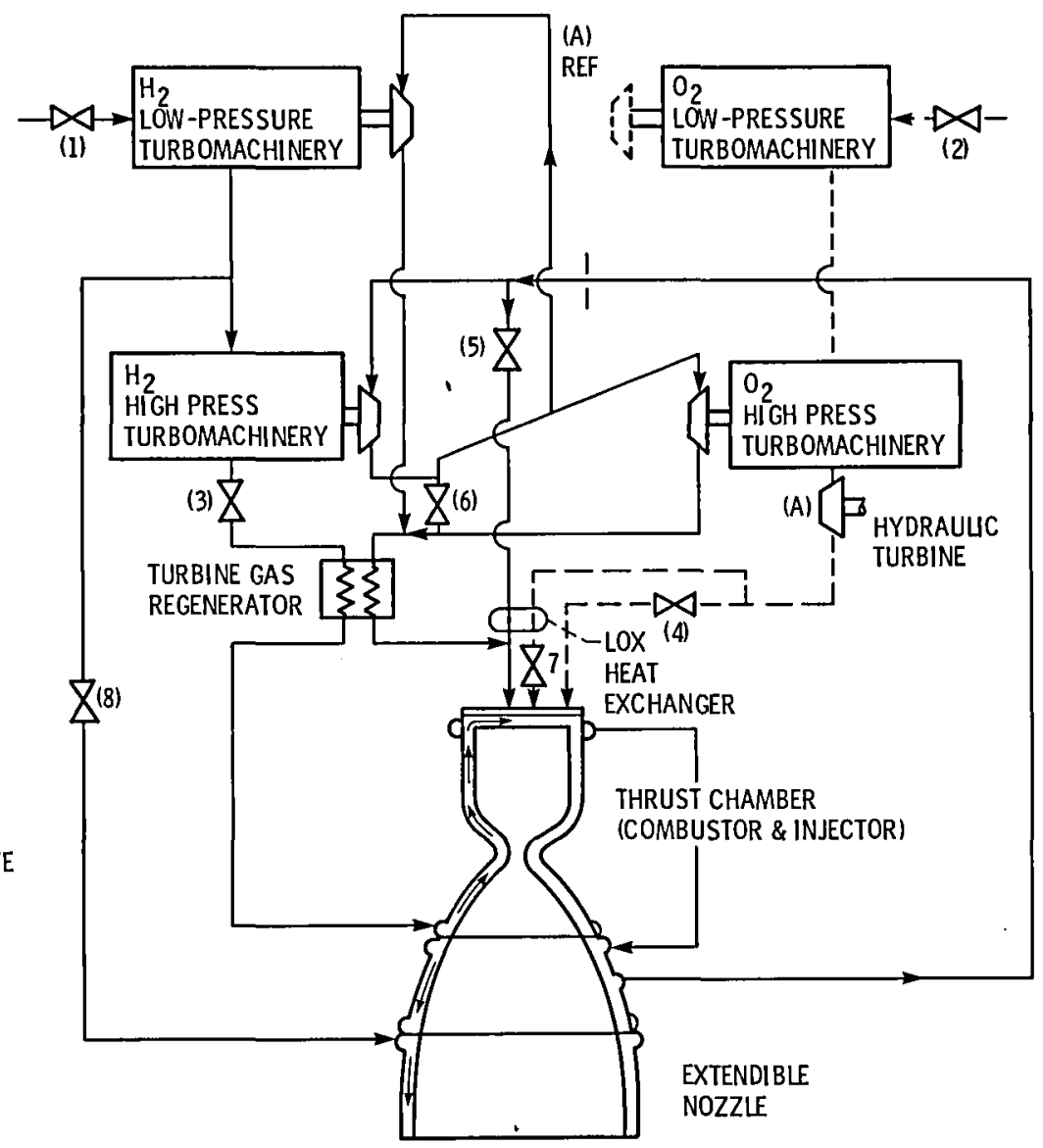

Figure 9. - Flow schematic; Rocketdyne advanced OTV propulsion concept. 


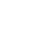




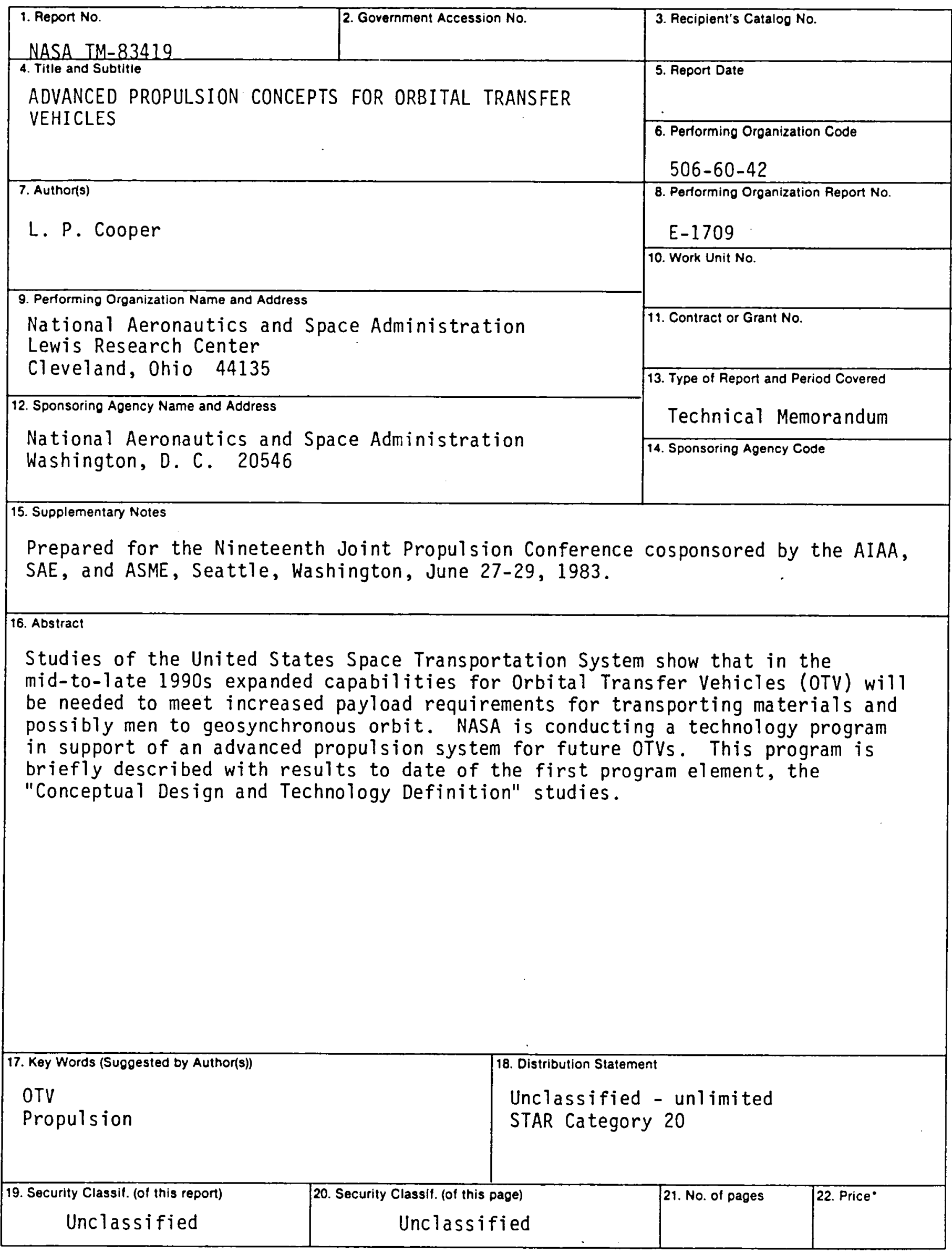

-For sale by the National Technical Information Service. Springfield. Virginia 22161 


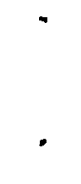


National Aeronautics and Space Administration

Washington, D.C.

20546

Official Business

Penalty for Private Use, $\$ 300$
SPECIAL FOURTH CLASS MAIL BOOK
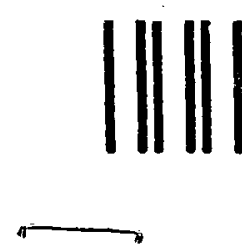

3

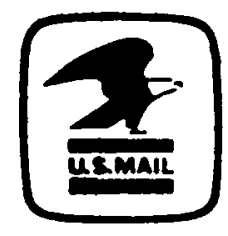

Postage and Fees Paid National Aeronautics and Spece Administration NASA-451

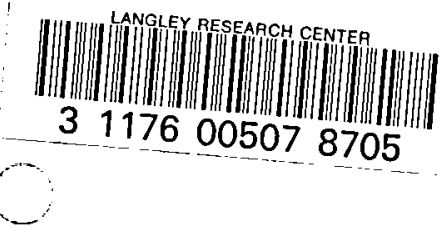

